\title{
Réactualisation des données sur la répartition des glossines au Mali
}

\author{
A. Djiteye ${ }^{1}$ S.K. Moloo ${ }^{2}$ K. Foua Bi ${ }^{3}$ \\ M. Touré ${ }^{2}$ S. Boiré ${ }^{1}$ S. Bengaly ${ }^{1}$ E. Coulibaly ${ }^{1}$

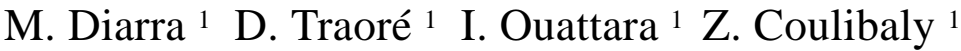

\section{Mots-clés}

Glossina morsitans submorsitans G lossina palpalis gambiensis - G lossina tachinoides - Distribution géographique - Densité de population Mali.

\section{Résumé}

L'aire de répartition des glossines au Mali couvre environ $200000 \mathrm{~km}^{2}$ au sud du parallèle $14^{\circ} 30^{\prime} \mathrm{N}$ et à l'ouest du méridien $4^{\circ} 30^{\prime} \mathrm{O}$. Q uatre espèces ont été signalées : deux riveraines ( $G$ lossina palpalis gambiensis et $G$. tachinoides) et deux de savane (G. morsitans submorsitans et $G$. longipalpis). G. morsitans submorsitans était répartie de manière plus ou moins continue le long des frontières avec la Côte d'Ivoire, la Guinée et le Sénégal jusqu'à la limite nord du parc national de la Boucle du Baoulé. A l'est de Bamako, la densité des populations était faible, apparemment discontinue dans les zones forestières. G. palpalis gambiensis était localisée le long de la rivière Bani, du fleuve Niger et de ses affluents, et des affluents du fleuve Sénégal (Baoulé, Bafing et Bagoé). $G$. tachinoides était répandue le long de la plupart des rivières et des grands cours d'eau de la partie sud-est du pays. Les prospections récentes n'ont pas revélé la présence de $\mathrm{G}$. Iongipalpis au Mali. Après plusieurs années de sécheresse et/ou un défrichement intensif, une diminution relativement importante de l'aire de répartition des glossines dans le pays a été constatée.

\section{INTRODUCTION}

Le Mali, enclavé au cœur de l'Afrique occidentale, a une superficie de $1241238 \mathrm{~km}^{2}$ pour une population de 8831713 habitants en 1994, soit une densité de 7,1 habitants $/ \mathrm{km}^{2}$.

Il peut être divisé en trois zones distinctes : la zone désertique au nord de l'isohyète $200 \mathrm{~mm}$ ( 50 p. 100 de la superficie totale), la zone sahélienne à l'est et au centre (25 p. 100 du territoire national) et la zone soudanienne à l'ouest et au sud (25 p. 100 du territoire national, avec une pluviométrie supérieure à $800 \mathrm{~mm}$ par an) (7) (figure 1). L'élevage constitue une activité économique importante du pays, mais ce secteur a été gravement affecté par une sécheresse qui sévit depuis plus d'une dizaine d'années. De 1982 à 1995, l'effectif du cheptel est passé de 6663000 à 5708000 têtes

1. Laboratoire Central Vétérinaire, BP 2295, Bamako, Mali

2. ILRI, PO Box 30709, Nairobi, Kenya

3. FAST, Université d'Abidjan, 22 BP 582, Abidjan 22, Côte d'Ivoire pour les bovins et de 12437000 à 13179000 pour les ovins-caprins. Le bétail est composé de 84 p. 100 de zébus et 16 p. 100 de taurins (trypanotolérants).

On assiste de plus en plus à une migration du cheptel vers le sud. En effet, dans les régions du Nord (Gao, Tombouctou et Mopti), il n'existait que 42,9 p. 100 du capital bovin en 1983, alors qu'en 1970 (c'est-à-dire avant la sécheresse de 1972-1973) ces mêmes régions hébergeaient 58,5 p. 100 du cheptel bovin national. L'expansion la plus importante de la production animale devra donc concerner la zone méridionale subhumide, là où de riches pâturages sont inexploités à cause de l'infestation par les glossines vectrices de trypanosomoses.

Les trypanosomes pathogènes rencontrés chez le bétail dans les zones infestées par les glossines au Mali sont : Trypanosoma congolense, $T$. vivax et $T$. brucei brucei. La sous-espèce $T$. brucei evansi est rencontrée chez les dromadaires hors de la zone infestée par les glossines ; elle est transmise par des vecteurs mécaniques (4). 


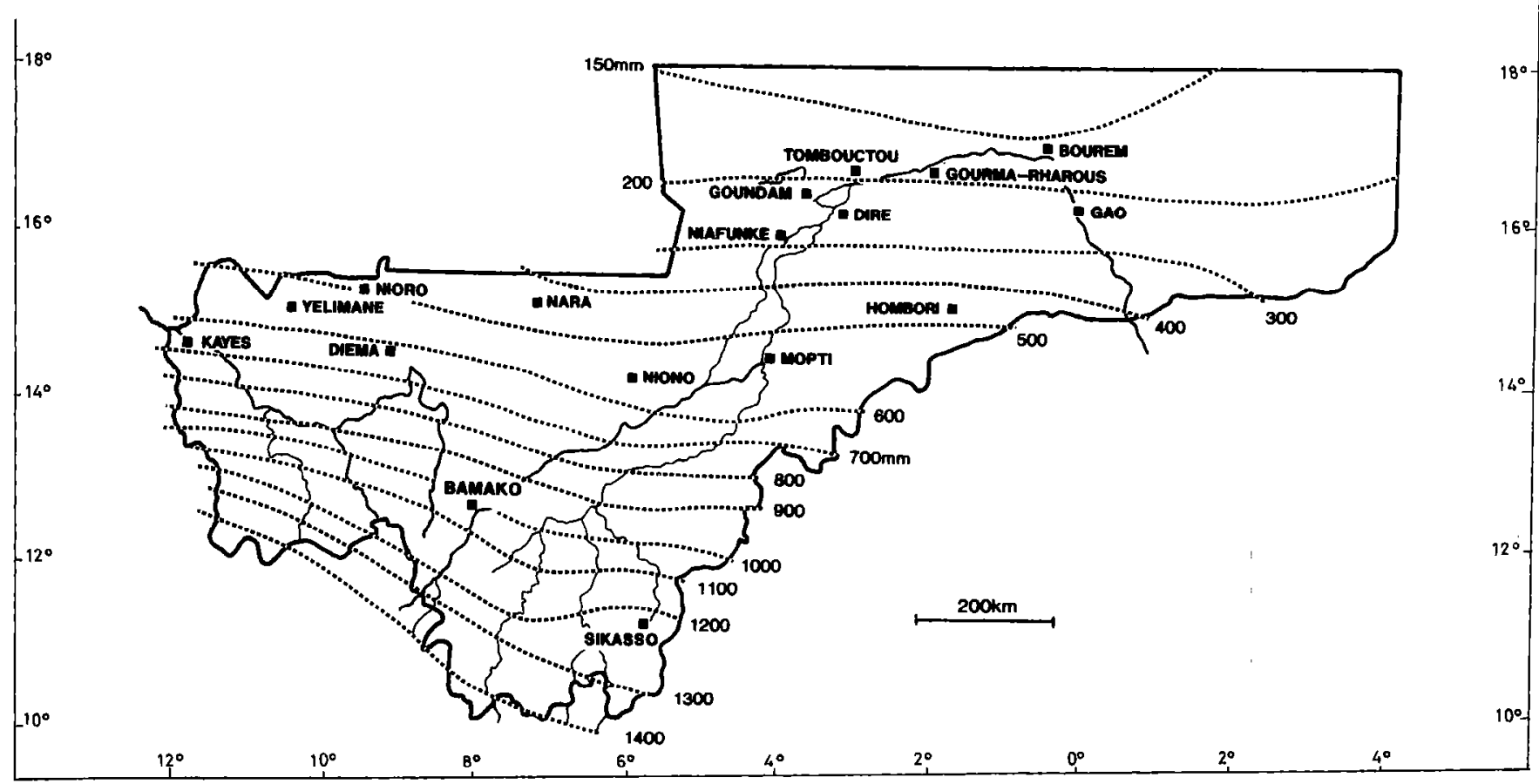

Figure 1 : carte des précipitations moyennes annuelles au Mali entre 1922 et 1980 (d'après le projet Barrière verte au Mali, 1989).

Environ 2,7 millions de bovins sont exposés au risque de la trypanosomose au Mali. La répartition de la maladie est assez bien connue, mais sa prévalence ne l'est pas par manque d'un réseau de diagnostic sur le terrain. Il est donc difficile d'évaluer, même approximativement, les pertes directes et indirectes causées par la trypanosomose animale au Mali (5).

Etant donné l'absence d'enquêtes systématiques, la situation de la trypanosomose humaine due à $T$. brucei gambiense est également mal connue. Toutefois, une recrudescence de la maladie du sommeil dans d'anciens foyers (cercles de Bougouni et Yanfolila) a été signalée ces dernières années, suite à un relâchement de la surveillance épidémiologique (3).

Le stade préliminaire avant toute intervention contre cette maladie consisterait à établir une carte générale et à jour de la répartition des mouches tsé-tsé. La section Entomologie du Laboratoire central vétérinaire (LCV), dans le cadre du Projet sectoriel de l'élevage au Mali (USAID), a mené des prospections afin de réactualiser les données sur la distribution des glossines au Mali.

\section{MATERIEL ET METHODES}

Pour les différents sondages, des cartes au $1 / 200000^{e}$ ont été utilisées (au total 28 cartes couvrent l'aire de répartition des glossines au Mali). Chaque carte recouvre un degré carré $\left(12100 \mathrm{~km}^{2}\right)$ et a été divisée en quatre secteurs (nord-est, nord-ouest, sud-est et sudouest) représentant chacun 0,25 degré carré $(55 \mathrm{~km} \times 55 \mathrm{~km})$.

Le piège biconique bleu Challier-Laveissière a été utilisé. Pendant les enquêtes (saison sèche en général), les pièges ont été placés au niveau des gîtes potentiels, à des endroits dégagés et durant la période d'activité des mouches : posés entre 7 ct $8 \mathrm{~h}$ et retirés entre $17 \mathrm{~h} 30$ et $18 \mathrm{~h} 30$. L'équipe de prospection utilisait très souvent un guide (chasseur, pêcheur ou éleveur) recruté sur place et connaissant parfaitement le terrain.
L'objectif étant de préciser très finement les cas limites de répartition des différentes espèces, les efforts ont donc été concentrés sur les zones « douteuses » où la présence des glossines n'était pas clairement établie.

Au niveau de chaque secteur (zone unité d'enquête) les renseignements suivants ont été notés :

- présence ou absence de glossines ;

- limites de répartition;

- densités apparentes (nombre de glossines par piège et par jour).

\section{RESULTATS ET DISCUSSION}

Quatre espèces de glossines, Glossina morsitans submorsitans, G. palpalis gambiensis, $G$. tachinoides ct $G$. longipalpis, avaient été signalées antérieurement au Mali $(1,8,9,10,11,13)$. Le tableau I montre la densité apparente des différentes espèces par carte et par secteur.

\section{Glossina morsitans submorsitans}

A l'ouest de l'axe Bamako-Bougouni, l'espèce de savane semblait indiquer une répartition plus on moins continue le long des frontières avec la Côte d'Ivoire, la Guinée et le Sénégal jusqu'à la limite nord du parc national de la Boucle du Baoulé (figure 2). A l'est de Bamako, seules des populations de densité faible, apparemment discontinue dans les zones forestières limitées telle que la forêt classée de la Faya, ont été trouvées (1).

Ces prospections ont revélé :

- son absence sur les cartes au 1/200 000 de Sandaré, Diéma,

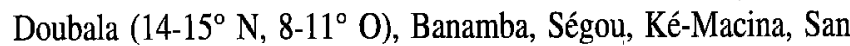
$\left(13-14^{\circ} \mathrm{N}, 4-8^{\circ} \mathrm{O}\right)$ et Yorosso $\left(12-13^{\circ} \mathrm{N}, 4-5^{\circ} \mathrm{O}\right)$; 


\section{Tableau I}

D ensité apparente des différentes espèces de glossines par carte et par secteur (résumé des prospections entre 1985 et 1989)

\begin{tabular}{|c|c|c|c|c|c|c|c|}
\hline \multicolumn{2}{|c|}{ Carte $1 / 200000^{e}$} & \multirow[t]{2}{*}{ Secteur } & \multicolumn{3}{|c|}{ D ensité apparente } & \multirow[t]{2}{*}{ Cours d'eau } & \multirow[t]{2}{*}{ Localités } \\
\hline (période) & coord. & & G. $\mathbf{m}$. & G. p. & G. t. & & \\
\hline $\begin{array}{l}\text { Doubala } \\
\text { (mars-avr. 88) }\end{array}$ & $\begin{array}{l}14-15^{\circ} \mathrm{N} \\
08-09^{\circ} \mathrm{O}\end{array}$ & sud-ouest & 0 & 0 & 0 & $\begin{array}{l}\text { Baoulé/M indaba } \\
\text { Simbalo }\end{array}$ & $\begin{array}{l}\text { Foulabougou, } \\
\text { Bakorobougou, }\end{array}$ \\
\hline $\begin{array}{l}\text { Diéma } \\
\text { (mars-avr. 88) }\end{array}$ & $\begin{array}{l}14-15^{\circ} \mathrm{N} \\
09-10^{\circ} \mathrm{O}\end{array}$ & sud-est & 0 & 0 & 0 & Baoulé & $\begin{array}{l}\text { Gomborondia et } \\
\text { Djontèguèda }\end{array}$ \\
\hline $\begin{array}{l}\text { Kayes } \\
\text { (déc. 88) }\end{array}$ & $\begin{array}{l}14-15^{\circ} \mathrm{N} \\
11-12^{\circ} \mathrm{O}\end{array}$ & $\begin{array}{l}\text { sud-ouest } \\
\text { sud-est }\end{array}$ & $\begin{array}{l}0,6 \\
0\end{array}$ & $\begin{array}{l}0 \\
0\end{array}$ & $\begin{array}{l}0 \\
0\end{array}$ & $\begin{array}{l}\text { Sanakolé } \\
\text { Sénégal, Papara }\end{array}$ & $\begin{array}{l}\text { Kayes, Faga, Sébétou } \\
\text { Balandougou, Diamou }\end{array}$ \\
\hline $\begin{array}{l}\text { San } \\
\text { (jan.-avr. 86) }\end{array}$ & $\begin{array}{l}13-14^{\circ} \mathrm{N} \\
04-05^{\circ} \mathrm{O}\end{array}$ & $\begin{array}{l}\text { nord-ouest } \\
\text { nord-est } \\
\text { sud-ouest } \\
\text { sud-est }\end{array}$ & $\begin{array}{l}0 \\
0 \\
0 \\
0\end{array}$ & $\begin{array}{l}0,2 \\
0 \\
0,2 \\
0\end{array}$ & $\begin{array}{l}0 \\
0 \\
0 \\
0\end{array}$ & $\begin{array}{l}\text { Bani } \\
\text { Bamakô, Diama } \\
\text { Bani } \\
\text { Vohin, Sonikô }\end{array}$ & $\begin{array}{l}\text { Barmandougou, Bina } \\
\text { Fangasso, Mapè, Bonani } \\
\text { San, Sienso } \\
\text { Koula, Bénèna }\end{array}$ \\
\hline $\begin{array}{l}\text { Ké-Macina } \\
\text { (avr.-juin 86) }\end{array}$ & $\begin{array}{l}13-14^{\circ} \mathrm{N} \\
05-06^{\circ} \mathrm{O}\end{array}$ & $\begin{array}{l}\text { nord-ouest } \\
\text { nord-est } \\
\text { sud-ouest } \\
\text { sud-est }\end{array}$ & $\begin{array}{l}0 \\
0 \\
0 \\
0\end{array}$ & $\begin{array}{l}0 \\
0 \\
0,28 \\
0,80\end{array}$ & $\begin{array}{l}0 \\
0 \\
0 \\
0\end{array}$ & $\begin{array}{l}\text { Niger } \\
\text { Niger } \\
\text { Bani } \\
\text { Bani }\end{array}$ & $\begin{array}{l}\text { Sansanding, Sibila, Sossé } \\
\text { Ké-Macina, M iérou } \\
\text { Cinzana, Douna } \\
\text { N'Goan, Tabara }\end{array}$ \\
\hline $\begin{array}{l}\text { Ségou } \\
\text { (juin 86) }\end{array}$ & $\begin{array}{l}13-14^{\circ} \mathrm{N} \\
06-07^{\circ} \mathrm{O}\end{array}$ & nord-est & 0 & 0 & 0 & Niger & Markala, Banankoro \\
\hline $\begin{array}{l}\text { Banamba } \\
\text { (mai-juin 88) }\end{array}$ & $\begin{array}{l}13-14^{\circ} \mathrm{N} \\
07-08^{\circ} \mathrm{O}\end{array}$ & $\begin{array}{l}\text { nord-ouest } \\
\text { nord-est } \\
\text { sud-ouest } \\
\text { sud-est }\end{array}$ & $\begin{array}{l}0 \\
0 \\
0 \\
0\end{array}$ & $\begin{array}{l}0 \\
0 \\
0 \\
2,20\end{array}$ & $\begin{array}{l}0 \\
0 \\
0 \\
0\end{array}$ & $\begin{array}{l}\text { Kolossa } \\
\text { Diawolo, } \\
\text { Dionokô } \\
\text { Koblé } \\
\text { Niger }\end{array}$ & $\begin{array}{l}\text { Batamobougou, Banankoro } \\
\text { Niabougou, Kondo } \\
\text { Koula, Tiékourabougou } \\
\text { Kondioukou, Nyamina }\end{array}$ \\
\hline $\begin{array}{l}\text { Kolokani } \\
\text { (avr. 88) } \\
\text { (juil. 89) }\end{array}$ & $\begin{array}{l}13-14^{\circ} \mathrm{N} \\
08-09^{\circ} \mathrm{O}\end{array}$ & $\begin{array}{l}\text { nord-ouest } \\
\text { nord-est } \\
\text { sud-ouest } \\
\text { sud-est }\end{array}$ & $\begin{array}{l}1^{*} \\
0 \\
0,2 \\
0\end{array}$ & $\begin{array}{l}0 \\
0,45 \\
0,10 \\
0\end{array}$ & $\begin{array}{l}0 \\
0 \\
0 \\
0\end{array}$ & $\begin{array}{l}\text { Kénié } \\
\text { Baoulé } \\
\text { Baoulé, Kénié } \\
\text { Dlani }\end{array}$ & $\begin{array}{l}\text { Madina, Kolé, Léko } \\
\text { Missira, Konibida } \\
\text { Madina, Forêt de Fina } \\
\text { Faladiè, Kondjiribougou }\end{array}$ \\
\hline $\begin{array}{l}\text { Kita } \\
\text { (avr. 88) } \\
\text { (juin 89) }\end{array}$ & $\begin{array}{l}13-14^{\circ} \mathrm{N} \\
09-10^{\circ} \mathrm{O}\end{array}$ & $\begin{array}{l}\text { nord-ouest } \\
\text { nord-est } \\
\text { sud-ouest } \\
\text { sud-est }\end{array}$ & $\begin{array}{l}0,2 \\
0 \\
0,20 \\
0,10\end{array}$ & $\begin{array}{l}0 \\
0 \\
1,80 \\
0\end{array}$ & $\begin{array}{l}0 \\
0\end{array}$ & $\begin{array}{l}\text { Baoulé, } \\
\text { Badinnkô } \\
\text { Baoulé, } \\
\text { Keniébakô } \\
\text { Bakoye } \\
\text { Badinnkô }\end{array}$ & $\begin{array}{l}\text { Toukoto, Kourininkoto } \\
\text { Kourinikoto, Faréna } \\
\text { Kita, Djélikébafata } \\
\text { Kita, Makodi, Kouroundi }\end{array}$ \\
\hline $\begin{array}{l}\text { Bafoulabé } \\
\text { (jan. 89) }\end{array}$ & $\begin{array}{l}13-14^{\circ} \mathrm{N} \\
10-11^{\circ} \mathrm{O}\end{array}$ & $\begin{array}{l}\text { nord-ouest } \\
\text { sud-ouest } \\
\text { sud-est }\end{array}$ & $\begin{array}{l}0,20 \\
0,25 \\
2\end{array}$ & $\begin{array}{l}0 \\
0,12 \\
-\end{array}$ & $\begin{array}{l}0 \\
0 \\
-\end{array}$ & $\begin{array}{l}\text { Sénégal, Bakoye } \\
\text { Bafing } \\
\text { Bafing }\end{array}$ & $\begin{array}{l}\text { Bafoulabé, For-Dioubéba } \\
\text { Manantali, Bangassi } \\
\text { Manantali }\end{array}$ \\
\hline $\begin{array}{l}\text { Kossanto } \\
\text { (déc. 88) }\end{array}$ & $\begin{array}{l}13-14^{\circ} \mathrm{N} \\
11-12^{\circ} \mathrm{O}\end{array}$ & $\begin{array}{l}\text { nord-ouest } \\
\text { nord-est } \\
\text { sud-ouest } \\
\text { sud-est }\end{array}$ & $\begin{array}{l}2,66 \\
5,33 \\
5,60 \\
1\end{array}$ & $\begin{array}{l}0 \\
- \\
6,50 \\
21\end{array}$ & $\begin{array}{l}0 \\
- \\
0 \\
0\end{array}$ & $\begin{array}{l}\text { Kamaya } \\
\text { - } \\
\text { Falémé } \\
\text { Manakoto }\end{array}$ & $\begin{array}{l}\text { Bassé,Bafoulabé,Bourdala } \\
\text { Dialafara, Kabélea } \\
\text { Gourbassi } \\
\text { Dialafara, Manakoto }\end{array}$ \\
\hline $\begin{array}{l}\text { Keniéba } \\
\text { (jan. 89) }\end{array}$ & $\begin{array}{l}12-13^{\circ} \mathrm{N} \\
11-12^{\circ} \mathrm{O}\end{array}$ & $\begin{array}{l}\text { nord-est } \\
\text { sud-est }\end{array}$ & $\begin{array}{l}0,75 \\
13,6\end{array}$ & $\begin{array}{l}0,10 \\
4,40\end{array}$ & $\begin{array}{l}0 \\
0\end{array}$ & $\begin{array}{l}\text { Doundi } \\
\text { Kassaya }\end{array}$ & $\begin{array}{l}\text { Kéniéba, Linguékoto } \\
\text { Faléa, Rép. de Guinée }\end{array}$ \\
\hline $\begin{array}{l}\text { Yorosso } \\
\text { (nov. 85) }\end{array}$ & $\begin{array}{l}12-13^{\circ} \mathrm{N} \\
04-05^{\circ} \mathrm{O}\end{array}$ & $\begin{array}{l}\text { nord-ouest } \\
\text { sud-ouest }\end{array}$ & $\begin{array}{l}0 \\
0\end{array}$ & $\begin{array}{l}0 \\
0\end{array}$ & $\begin{array}{l}0 \\
0\end{array}$ & $\begin{array}{l}\text { Banifing } \\
\text { Kilé }\end{array}$ & $\begin{array}{l}\text { Kimparana, Kifa } \\
\text { Mahou, Yorosso, Boura }\end{array}$ \\
\hline
\end{tabular}


Tableau I (suite)

Densité apparente des différentes espèces de glossines par carte et par secteur (résumé des prospections entre 1985 et 1989)

\begin{tabular}{|c|c|c|c|c|c|c|c|}
\hline \multicolumn{2}{|c|}{ Carte $1 / 200000^{e}$} & \multirow[t]{2}{*}{ Secteur } & \multicolumn{3}{|c|}{ D ensité apparente } & \multirow[t]{2}{*}{ Cours d'eau } & \multirow[t]{2}{*}{ Localités } \\
\hline (période) & coord. & & G. $\mathbf{m}$. & G. p. & G. t. & & \\
\hline $\begin{array}{l}\text { Koutiala } \\
\text { (nov. 85) } \\
\text { (jan.-fév. 86) }\end{array}$ & $\begin{array}{l}12-13^{\circ} \mathrm{N} \\
05-06^{\circ} \mathrm{O}\end{array}$ & $\begin{array}{l}\text { nord-ouest } \\
\text { nord-est } \\
\text { sud-ouest } \\
\text { sud-est }\end{array}$ & $\begin{array}{l}0 \\
0 \\
0,07 \\
0\end{array}$ & $\begin{array}{l}0,40 \\
0 \\
2,60 \\
4\end{array}$ & $\begin{array}{l}0 \\
0 \\
5,10 \\
0,66\end{array}$ & $\begin{array}{l}\text { Pékadouzou } \\
\text { Fawara } \\
\text { Banifing } \\
\text { Kapakô,Tarasso }\end{array}$ & $\begin{array}{l}\text { N'Péssoba, N'Tarla } \\
\text { Kounia } \\
\text { Zangasso } \\
\text { Koutiala, Karangasso }\end{array}$ \\
\hline $\begin{array}{l}\text { Dioila } 1 \\
\text { (juin } 89 \text { ) }\end{array}$ & $\begin{array}{l}2-13^{\circ} \mathrm{N} \\
06-07^{\circ} \mathrm{O}\end{array}$ & nord-est & 0 & 1,60 & 0 & $\begin{array}{l}\text { Bagoé } \\
\text { Banifing }\end{array}$ & $\begin{array}{l}\text { Dambalafara, Kona, } \\
\text { N'Golobougou }\end{array}$ \\
\hline $\begin{array}{l}\text { Bamako-Est } \\
\text { (juil. 89) }\end{array}$ & $\begin{array}{l}12-13^{\circ} \mathrm{N} \\
07-08^{\circ} \mathrm{O}\end{array}$ & nord-est & 0 & 21,7 & 0 & Niger & $\begin{array}{l}\text { Forêt classée de Tienfala } \\
\text { Forêt classée de la Faya }\end{array}$ \\
\hline $\begin{array}{l}\text { Massigui } \\
\text { (oct. 85) }\end{array}$ & $\begin{array}{l}11-12^{\circ} \mathrm{N} \\
06-07^{\circ} \mathrm{O}\end{array}$ & $\begin{array}{l}\text { nord-ouest } \\
\text { nord-est } \\
\text { sud-est }\end{array}$ & $\begin{array}{l}0 \\
0 \\
0\end{array}$ & $\begin{array}{l}0,20 \\
1,40 \\
0\end{array}$ & $\begin{array}{l}0 \\
0,50 \\
0\end{array}$ & $\begin{array}{l}\text { Kôba } \\
\text { Kôba } \\
\text { Outénébougoukô }\end{array}$ & $\begin{array}{l}\text { Sanso, M orila } \\
\text { Blendio, Kona } \\
\text { N iéna, O uaténébougou }\end{array}$ \\
\hline
\end{tabular}

DAP : densité apparente $=$ nombre de glossines/piège/jour

* Capture sur véhicule

- : pas de piégeage dans le biotope indiqué

G. $m .=$ Glossina morsitans $;$ G. $p .=$ Glossina palpalis $;$ G. $t .=$ Glossina tachinoides

- son absence dans le secteur nord-ouest de la carte de Koutiala, mais sa présence dans le secteur sud-ouest (densité apparente très faible, un seul mâle a été capturé dans le véhicule des auteurs) ;

- sa présence dans le secteur sud-ouest de la carte de Kayes (vers Sébétou) et sur les cartes de Kossanto, Bafoulabé, Kita et Kolokani (limitée au nord par la latitude $14^{\circ} \mathrm{N}$ ).

\section{Glossina palpalis gambiensis}

Cette espèce riveraine était localisée (1) (figure 3) :

- le long de la rivière Bani et de ses affluents (Baoulé, Bagoé, Banifing), de la frontière sud du Mali jusqu'au niveau du village de Barmandougou (situé un peu au nord de la latitude $13^{\circ} 30^{\prime} \mathrm{N}$, environ à $65 \mathrm{~km}$ au nord-est de la ville de San) ;

- le long du fleuve Niger et de ses affluents, à partir de la frontière sud du Mali jusqu'à $60 \mathrm{~km}$ au sud-ouest de la ville de Ségou (située au niveau de la latitude $13^{\circ} 30^{\prime} \mathrm{N}$ ) ;

- le long des affluents du fleuve Sénégal (Falémé, Bafing, Bakoye, Baoulé).

Ces enquêtes récentes ont révélé :

- sa concentration le long de la rivière Bani, sur la carte de San $\left(13-14^{\circ} \mathrm{N}, 4-5^{\circ} \mathrm{O}\right)$;

- son absence sur la carte de Yorosso $\left(12-13^{\circ} \mathrm{N}, 4-5^{\circ} \mathrm{O}\right)$, et dans le secteur nord-est de la carte de Koutiala $\left(12^{\circ} 30^{\prime}-13^{\circ} \mathrm{N}\right.$, $\left.5-5^{\circ} 30^{\prime} \mathrm{O}\right)$;

- son absence sur les cartes de Kayes, Sandaré, Diéma et Doubala $\left(14-15^{\circ} \mathrm{N}, 8-12^{\circ} \mathrm{O}\right)$;
- son recul sur les cartes de Kossanto, Bafoulabé et Kita (absence dans les secteurs nord : $13^{\circ} 30^{\prime}-14^{\circ} \mathrm{N}, 9-12^{\circ} \mathrm{O}$; sa présence au sud de la latitude $13^{\circ} 30^{\prime} \mathrm{N}$, dans les secteurs sud) ;

- sa stabilisation sur la carte de Kolokani et le long de la rivière Baoulé.

\section{Glossina tachinoides}

Elle était répandue le long de la plupart des rivières et grands cours d'eau de la partie sud-est du pays (Sankarani, Baoulé, Bagoé, Banifing, etc.) (figure 4).

Cette seconde espèce riveraine avait manifesté un recul vers le sud, mais elle semblait se stabiliser lors de cette étude dans sa limite nord de 1989 , soit $13^{\circ} \mathrm{N}$ (1). En effet, des sondages récents ont permis de détecter des densités plus ou moins élevées dans les secteurs sud-est et sud-ouest de la carte de Koutiala.

\section{Glossina longipalpis}

Cette seconde espèce de savane avait été seulement signalée dans une petite zone le long de la frontière guinéenne, juste au nord du parallèle $12^{\circ} \mathrm{N}$ et à l'ouest du méridien $11^{\circ} \mathrm{O}$.

Au total 260 glossines (138 mâles et 122 femelles) du sous-genre Glossina (ancien groupe morsitans), capturées dans cette zone, ont été analysées à l'aide d'une loupe binoculaire. La recherche de soies sur le troisième article antennaire, l'observation des lobes médians des cerques chez les mâles et la taille des soies scutellaires médianes chez les femelles n'ont pas permis de révéler la présence de Glossina longipalpis durant cette étude non exhaustive. En effet, l'enquête effectuée en saison sèche froide n'a duré qu'une semaine (janvier 1989). 


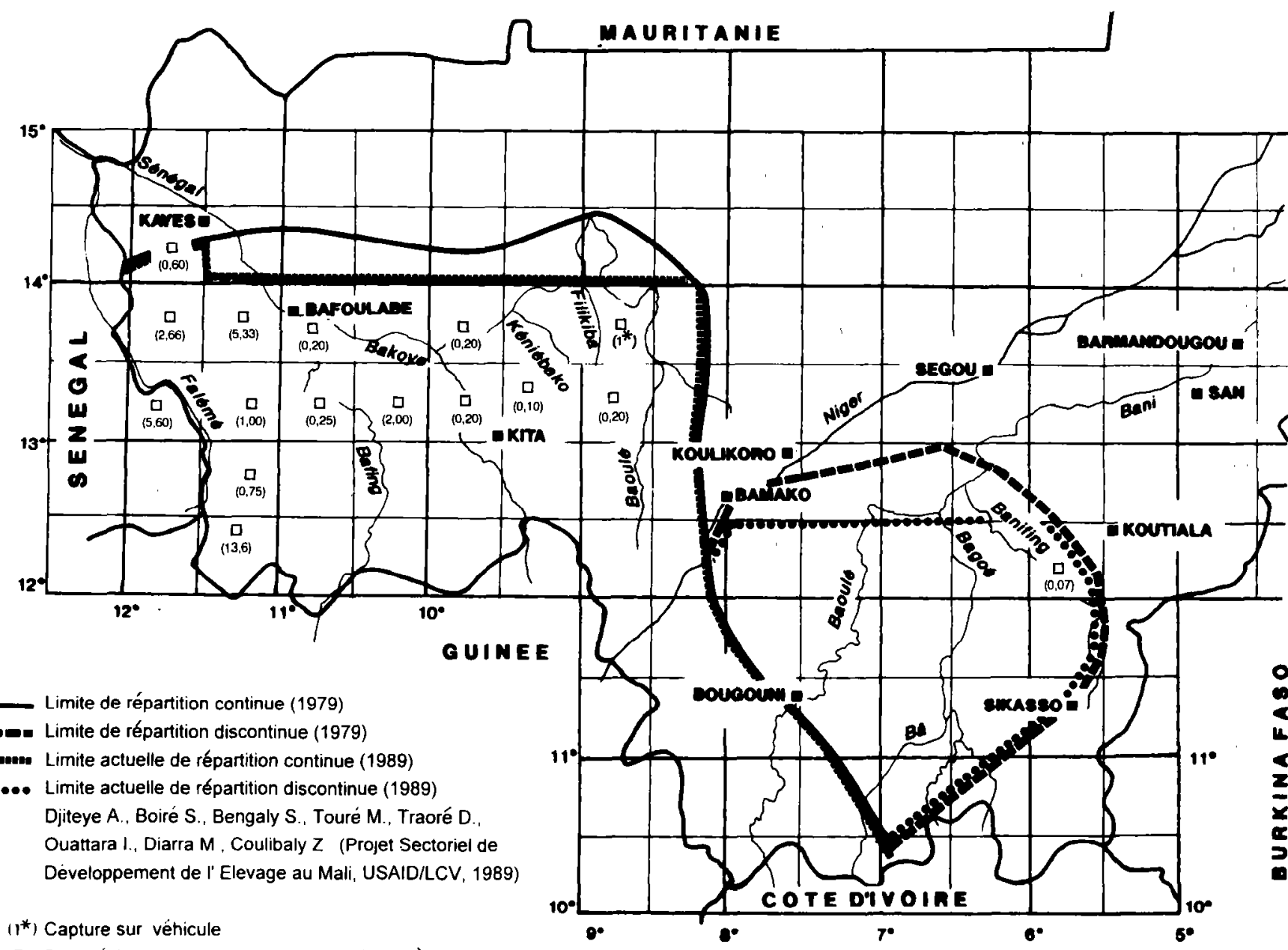

- Densité Apparente $=$ Nombre de glossines/piège/jour $(0,20)$

Figure 2 : carte de la limite nord de répartition de Glossina morsitans submorsitans au Mali.

\section{CONCLUSION}

Une diminution relativement importante de l'aire de répartition de G. palpalis gambiensis et de G. morsitans submorsitans a élé constatée, après plusieurs années de sécheresse (tarissement de cours d'eau permanents) et un défrichement intensif (notamment dans la zone de Koutiala pour la culture du coton, et le long du fleuve Sénégal et de ses affluents pour la culture maraîchère).

Le recul de ces deux espèces peut n'être que temporaire, car les auteurs estiment que le phénomène peut être réversible après rétablissement d'une pluviométrie normale, multiplication des barrages de retenue d'eau et intensification des reboisements. C'est ainsi que $G$. morsitans submorsitans, qui était absente dans les zones de Kita et de Kolokani en mars-mai 1988 (saison sèche), a élé retrouvée dans les mêmes zones en juin-juillet 1989, après la bonne pluviométrie de 1988 .

Le long des cours d'eau de la partie sud-est du pays, Glossina tachinoides, espèce riveraine reconnue plus apte à supporter la sécheresse, manifestait curieusement un recul plus rapide vers le sud que G. palpalis gambiensis. Sa stabilisation à la latitude $13^{\circ} \mathrm{N}$ comme limite nord de répartition, environ à $100 \mathrm{~km}$ au sud de celle de $G$. palpalis gambiensis, doit être affirmée avec prudence.

Les résultats obtenus durant cette étude non exhaustive donnent une idée générale des limites nord des aires de répartition des trois espèces de glossines plus ou moins largement répandues au Mali. Pour des études plus poussées relatives à la lutte anti-vectorielle, il est nécessaire d'affiner les méthodes de prospection, avec l'utilisation des attractifs olfactifs sur les pièges, pour la détection des populations résiduelles de glossines. L'association acétone/octénol augmente considérablement l'efficacité des pièges contre G. morsitans submorsitans ; les combinaisons acétone/octénol/meta-crésol, acétone/octénol/urine de vache et octénol/meta-crésol sont très efficaces contre $G$. tachinoides, et, dans une certaine mesure, contre G. palpalis gambiensis (6).

\section{Remerciements}

Nous tenons à remercier tous ceux qui nous ont permis de réaliser les enquêtes et la finition de ce travail, en particulier Dr O. Diall, Dr B. Seck, Dr C.F. Simbé, Dr B. Kouyaté, M. Z. Bocoum, M. B. Diarra, Y. Sanogo (LCV, Bamako), Dr R. Cook (APEX, USAID, Bamako), Pr P. Ranque (OMS, Genève), Pr A. Aouti, Dr M. Dagnogo (FAST, Abidjan), Dr U. Feldmann (Joint FAO/IAEA Division, Vienna), Dr M. Clair (IEMVT, MaisonsAlfort), Dr A. R. Gray, Dr R. Eley, M. J. Mwaura et M. F. Shikhubari (ILRI, Nairobi), Dr S. K. Katondo (OUA/IBAR), Dr S. M. Touré, Dr B. Bauer (CIRDES, Burkina Faso) et Dr R. de Deken (IMT, Belgique). Nos remerciements vont également à tous les agents du Service d'élevage, des Eaux et forêts qui nous ont chaque foís accueillis avec bienveillance et aidés dans nos entreprises. 


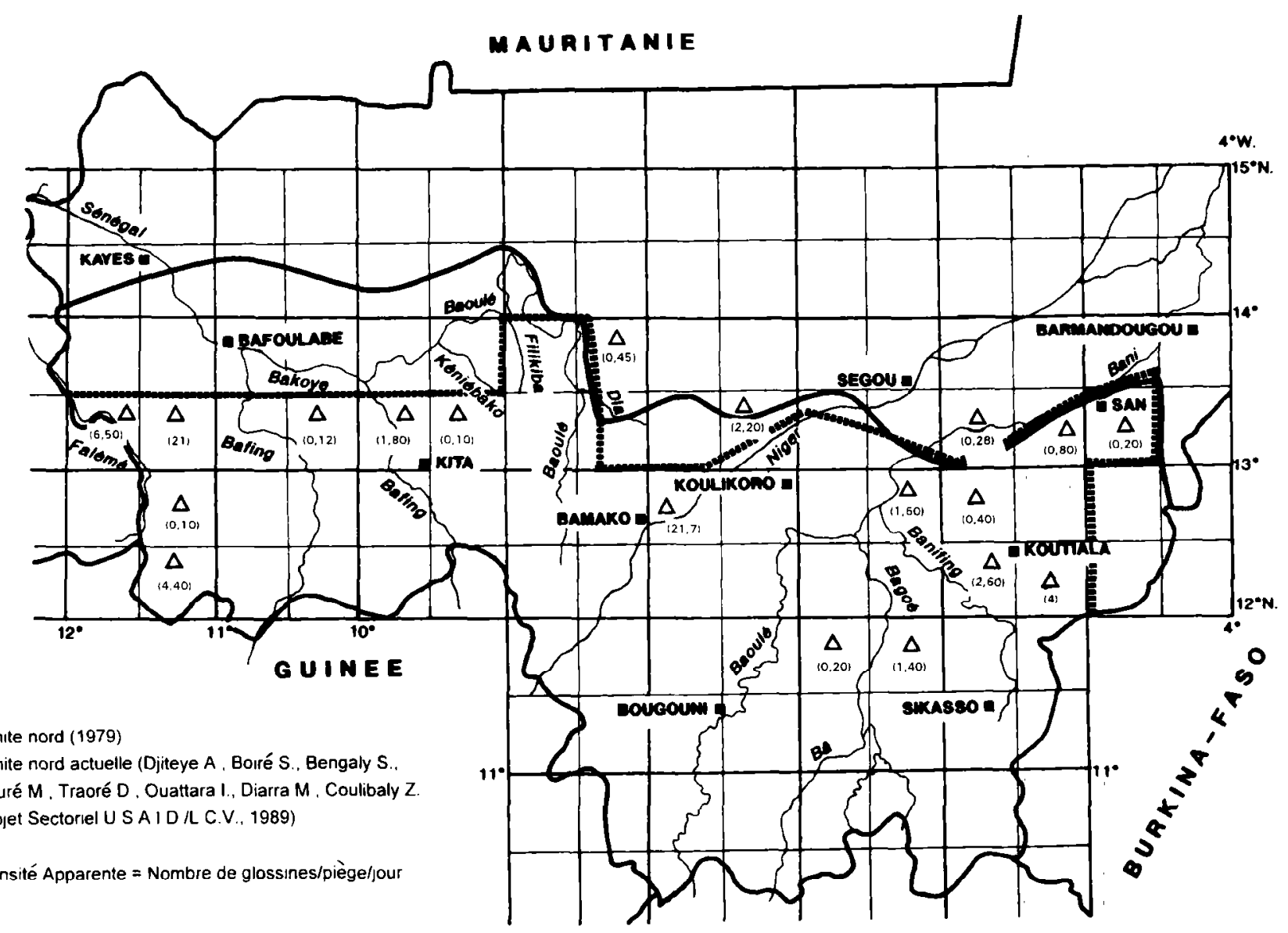

$(0,12)$

COTE D'I VORE

Figure 3 : carte de la limite nord de répartition de Glossina palpalis gambiensis au Mali.

\section{BIBLIOGRAPHIE}

1. ASHTON D.R., GOODWIN J.T., BA A., CISSE A., 1979. Répartition des mouches tsé-tsé en République du Mali. Texas, USA, Texas Agricultural Experiment Station/TAMU, p. 47-54. (Rapport intérimaire)

2. CLAIR M., DJITEYE A., 1988. Glossines et trypanosomes au Mali. In Atlas - Elevage et potentialités pastorales sahéliennes, synthèses cartographiques du Mali. Wageningen, Pays-Bas, CTA, Maisons-Alfort, France, IEMVT, p. 30-31.

3. DEMBELE P., DEGOGA 1., 1996. Programme national de lutte contre la trypanosomose humaine africaine au Mali. In : Réunion de coordination de lutte contre la trypanosomose, Abidjan, Côte d'lvoire, 7-9 mai 1996, 9 p.

4. DIALL O., 1993. Camel trypanosomosis in Mali. Contribution to the diagnosis and the epidemiology. Doctor of Science thesis, IMT, Anvers, Belgium, $92 \mathrm{p}$.

5. DJITEYE A., 1992. Projet de contrôle de la mouche tsé-tsé et de la trypanosomose en République du Mali. Bamako, Mali, Laboratoire Central Vétérinaire, 68 p. (Rapport de la phase préparatoire d'un projet de lutte dans les zones libérées de l'onchocercose au Mali)
6. DIITEYE A., GYENING K.O., AMSLER S., TRAORE D., SANOGO Y., 1995. Efficacité comparée de différentes associations d'odeurs sur les glossines, tabanides et stomoxes au Mali. In : $23^{\text {e }}$ CSIRLT, Banjul, Gambie, 11-15 septembre 1995.

7. DNSI, 1995. Bilan diagnostic du développement humain durable au Mali. Bamako, Mali, DNSI.

8. FORD 1., KATONDO K.M., 1973. Maps of tsetse flies distribution in Africa. Nairobi, Kenya, OUA/STRC, London, United Kingdom, Cook Hammond \& Kell.

9. KATONDO K.M., 1984. Tsetse distribution maps (an interim report). Insect Sci. Appl., 5 (5): 381-388.

10. MOLOO S.K., 1985. Distribution of Glossina species in Africa. Acta trop., 42: 275-281.

11. MOLOO S.K., 1993. The distribution of Glossina species in Africa and their hosts. Insect Sci. Appl., 14 (4): 511-527.

12. OKIWELU S.N., VAN WETTERE P., MAIGA S., BOUARE S., CRANS W., 1983. Contribution to the distribution of Glossina (Diptera: Glossinidae) in Mali. Bull. ent. Res., 71: 195-205.

13. RICKENBACH A., 1961. Carte de répartition des glossines en Afrique occidentale d'expression française. Bobo-Dioulasso, Haute Volta, ORSTOM, Centre MURAZ.

Reçu le 3.4.96, accepté le 24.7 .97 


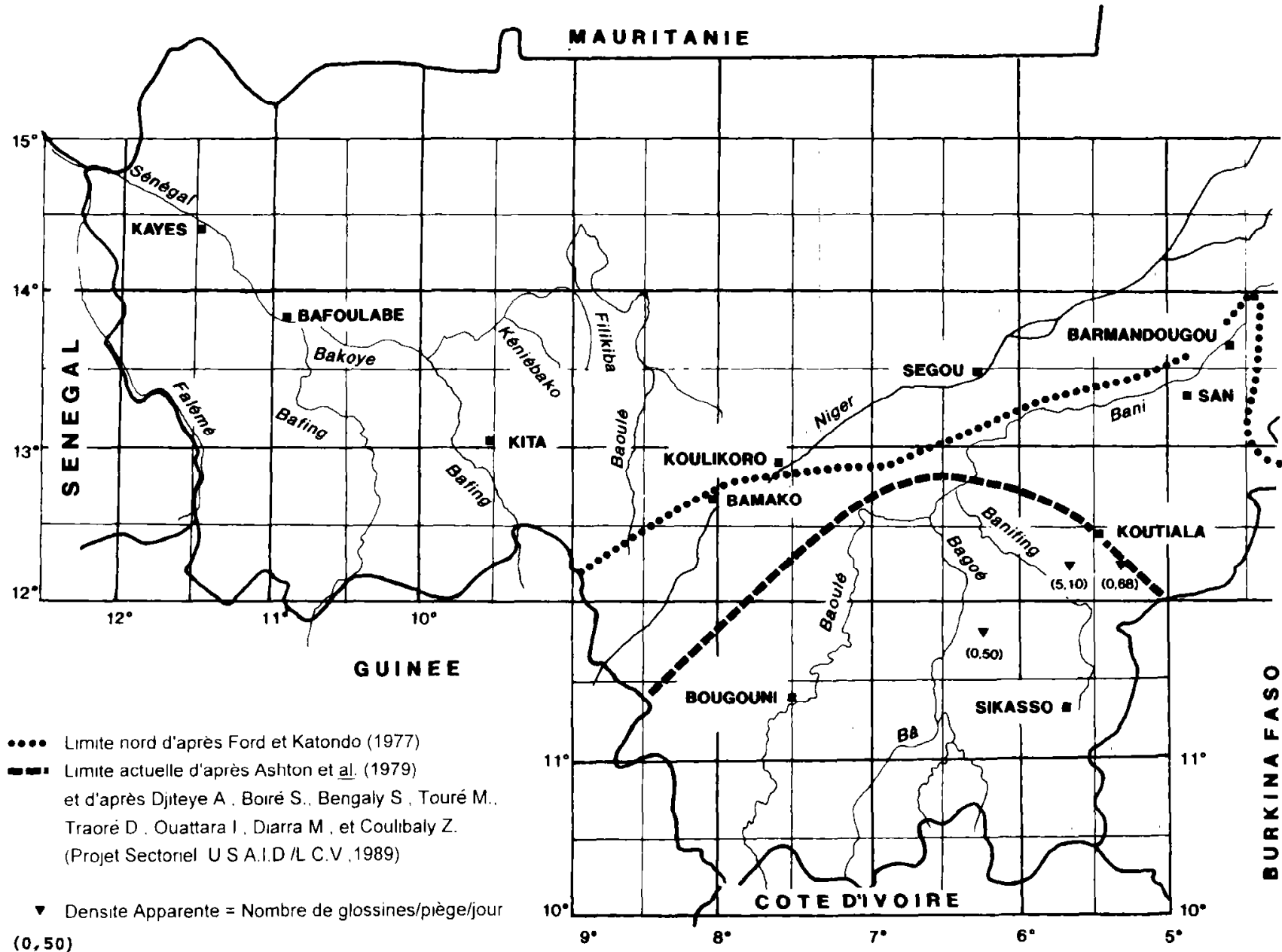

Figure 4 : carte de la limite nord de répartition de Glossina tachinoides au Mali.

\section{Summary}

Djiteye A., Moloo S.K., Foua Bi K., Touré M., Boiré S., Bengaly S., Coulibaly E., Diarra M., Traoré D., Ouattara I., Coulibaly Z. Update on the distribution of tsetse flies in Mali

The area of tsetse fly infestation in Mali is approximately $200,000 \mathrm{~km}^{2}$, South of parallel $14^{\circ} 30^{\prime} \mathrm{N}$ and West of meridian $4^{\circ} 30^{\prime} \mathrm{W}$. Four species have been reported: two riverine (Glossina palpalis gambiensis and $G$. tachinoides) and two savannah species (G. morsitans submorsitans and G. longipalpis). G. morsitans submorsitans has a more or less continuous distribution along the borders with Côte d'Ivoire, and Senegal up to the northern limit of the National Park Boucle du Baoulé. A low population density, apparently discontinuous in forest zones, has been encountered East of Bamako, in forest zones. G. palpalis gambiensis was localized along the Bani River, the Niger River and its tributaries, as well as tributaries of the Senegal River (Baoulé, Bafing and Bagoé). G. tachinoides was present in most of the riverine forest galleries in the South-Eastern part of the country. The presence in Mali of $G$. longipalpis was not confirmed during this survey. A relatively important decrease in the distribution zones of tsetse flies has been observed in Mali following several years of drought and/or an intensive deforestation.

Keys words: Glossina morsitans submorsitans - Glossina palpalis gambiensis - Glossina tachinoides - Geographical distribution - Population density - Mali.

\section{Resumen}

Djiteye A., Moloo S.K., Foua Bi K., Touré M., Boiré S., Bengaly S., Coulibaly E., Diarra M., Traoré D., Ouattara I., Coulibaly Z. Reactualización de los datos sobre la repartición de las glosinas en Malí

El área de repartición de las glosinas en Malí cubre alrededor $200000 \mathrm{~km}^{2}$ al sur del paralelo $14^{\circ} 30^{\prime} \mathrm{N}$ y al oeste delmeridiano $4^{\circ} 30^{\prime} \mathrm{O}$. Se señalan cuatro especies: dos ribereñas (Glossina palpalis gambiensis y $G$. tachinoides) y dos de sabana ( $G$. morsitans submorsitans y $G$. longipalpis). G. morsitans submorsitans presenta una repartición más o menos continua a lo largo de las fronteras con Costa de Marfil, Guinea y Senegal, hasta el límite norte del Parque nacional de la Boucle du Baoulé. Al este de Bamako, la densidad de las poblaciones fue baja y aparentemente discontinua en las zonas forestales. G. Palpalis gambiensis se localizó a lo largo del río Bani, del río Níger y de sus afluentes, de los afluentes del río Senegal (Baoulé, Bafing y Bagoé). G. tachinoides se encontró distribuída a lo largo de la mayor parte de los ríos y de los grandes lechos de agua de la parte sudeste del país. Las últimas inspecciones realizadas no revelaron la presencia de G. longipalpis en Malí. Debido a varios años de sequía y/o a un desmonte (o roza) intensivo, se observó una disminiución relativamente importante del área de repartición de las glosinas en el país.

Palabras clave: Glossina morsitans submorsitans - Glossina palpalis gambiensis - Glossina tachinoides - Distribución geográfica - Densidad de la población - Malí. 